\title{
AN ALGORITHM FOR CONTROLLING A TRACTION ASYNCHRONOUS DRIVE THAT MINIMIZES ELECTRICAL POWER LOSSES
}

\author{
Abit Burkhanhodjaev $^{1}$, Elena Iksar $^{2}$, Mokhira Idriskhodjaeva ${ }^{1}$ \\ ${ }^{1}$ Tashkent State Technical University, Universit-2 Tashkent, 100095, Uzbekistan \\ ${ }^{2}$ Tashkent institute of railway engineering, Adilkhodjayev - 1, Tashkent, 100167, Uzbekistan
}

\begin{abstract}
The article discusses the improvement of traction and energy performance main-line locomotives due to more complete use of possibilities of flexible microprocessor control and efficient algorithm that provides reduction of electrical losses in the traction asynchronous drive over the entire range working capacity. In the best way for a promising loco is tract with using a traction asynchronous drive. Such kind of tract drives allow completely use of the coupling mass of loco compares with collector track drive, due to the absence of a collector it is possible to increase the active length of the rotor and high reliability which makes it possible to realize increased axial power.
\end{abstract}

\section{Introduction}

Along with the forces generated in continuous mode and the coupling mass, the main traction and energy indicators of locomotives include such essential parameters as axial strength and energy losses over the whole speed range and load zone [1]. Based on the experience of industrialized countries, it can be concluded that the most preferable for promising locomotives is a drive using traction asynchronous motors. This type of engine allows the most complete use of the coupling mass of the locomotive in comparison with collector traction engines, due to the absence of a collector, it is possible to increase the active length of the rotor, and high reliability, which makes it possible to realize increased axial power. Experience in developing and testing samples of locomotives with traction asynchronous drive confirmed that the expected improvement in performance is possible if the system of automatic control of the traction electric drive is created, which ensures the implementation of all traction and power characteristics of the locomotive that can be achieved by using progressive three-phase traction motors.

\subsection{Methods}

As the operating experience shows, the locomotive works with partial loads for a significant part of the time zone [2]. When designing a traction motor, it is calculated for the minimum loss at rated power. At the same time, with a decrease in the realized power and efficiency of the engine, the relative electrical power loss decreases sharply and increases. The total time of operation of the electric drive in modes below the rated power is approximately $87-93 \%$ of the total time of operation in traction. In this regard it is proposed to use the principle of rational management to minimize losses the torque control time is determined by the time of changing the rotor flow coupling and is at least three rotor time constants, which for high-power ATDs is tenths of a second and can reach units of seconds. The area of traction characteristics of a mainline locomotive can be divided into two zones (Fig.1):

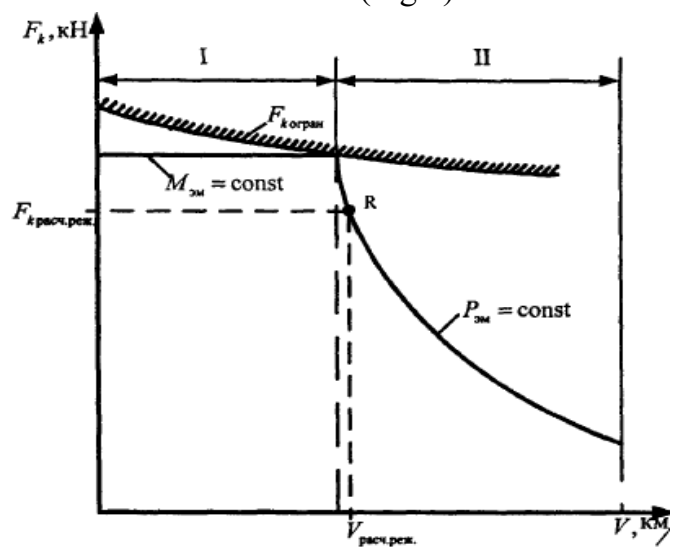

Fig.1 Traction characteristics of the locomotive

The first is the zone of maintaining a constant maximum torque $\mathrm{MEM}=$ const, it is used for starting in short modes. The second area overlays limit on the implemented capacity of the RAM = const. In this zone, there is a point that corresponds to the movement with 
the calculated speed R (V), which limits the minimum speed of movement in the long-term mode at a given power. In each zone of the traction characteristic, different requirements are imposed on the traction electric drive.

In the first case, it is necessary to maintain the maximum possible torque according to the coupling conditions, which are constantly changing depending on various factors, both external and internal. The total operating time in the first zone is not large compared to the second zone. The main indicator of performance in this zone is the speed of control of the MEM TAD, depending on changes in the coupling conditions. The main criterion for the operation of the locomotive traction drive in the first zone of maintaining constant torque is the increased rigidity of the characteristics of the traction engine. Unlike the first, the second zone is removed from the coupling restriction curve. Therefore, it does not require high rigidity characteristics as when working on the clutch limit. Traction and energy characteristics in this zone have a significant impact on energy consumption. Therefore, when working on a hyperbolic characteristic, it is most appropriate to use the control principle to minimize electrical power losses in the inverter-motor system. there are several ways to regulate an asynchronous traction motor: 1) regulation with a given stator voltage and constant absolute rotor slip; 2) regulation of an asynchronous traction motor with a given main thread coupling; 3) regulation of an asynchronous traction motor with a given stator thread coupling. The specified moment of the motor load is realized by the magnetic flux and the active component of the rotor current, the ratio of which determines the value of electric power losses in ad. There is a ratio between the magnetic flux and the rotor current, when the stator current becomes minimal at a constant load moment. To ensure this condition, it is necessary to select the appropriate values of the magnetic flux and frequency of the rotor current for each value of the load moment, depending on the saturation of the motor steel. The deviation of the frequency of the rotor current to smaller values leads to an increase in electric power loss in hell, and the deviation in a big way to the increase in the stator current due to the increase of the rotor current at small values of the magnetic flux, which also leads to increased electrical losses. To maintain a minimum of electrical power loss over a wide range of load moment values, it is necessary to increase the rotor current and magnetic flux as the motor load increases. For locomotives with asynchronous traction motors and static frequency converters, the most favorable mode of operation is at maximum efficiency of an asynchronous traction motor. It is known that the specified moment of the motor load is realized by the magnetic flux and the active component of the rotor current, the ratio of which determines the value of electric power losses in an asynchronous motor. There is a ratio between the magnetic flux and the rotor current, when the stator current becomes minimal at a constant load moment. To ensure this condition, it is necessary to select the appropriate values of the magnetic flux and the frequency of the rotor current for each value of the load moment, depending on the saturation of the motor steel. the deviation of the frequency of the rotor current to smaller values leads to an increase in electric power loss in hell, and the deviation in a big way - to an increase in the stator current due to the increase of the rotor current at small values of the magnetic flux, which also leads to increased electrical losses. to maintain a minimum of electrical power loss over a wide range of load moment values, it is necessary to increase the rotor current and magnetic flux as the motor load increases. To determine the minimum electrical power loss at a given frequency and varying the values of $\mathrm{u}$ and $\omega$, it is proposed to use the $\mathrm{C}$ calculation algorithm shown in Fig. 2. The initial data for the calculation is the specified power of the RAM, the actual speed of the RAM. These parameters determine the required torque of the asynchronous motor. In cases where it is possible to choose the mode of operation of the locomotive, it is necessary to take into account its efficiency or use the proposed control system that allows you to reduce losses when working at partial capacities because the locomotive operates at partial loads. The traction motor is calculated for a minimum of electrical losses for power values close to the nominal, while with a decrease in power, the efficiency of the engine decreases sharply, as the relative value of electrical power losses increases. If the moment is greater than the limit, the signal is sent to the vector control system If the moment is less than or equal to the limit, then the signal for the specified moment is sent to the control system to minimize electrical losses. The first is the zone of maintaining a constant maximum torque MEM = const, it is used for starting in short modes. The second area imposes a limit on the implemented power of $\mathrm{RAM}=$ const. In this zone, there is a point that corresponds to the movement with the calculated R (VSpeed, F-speed dir), which limits the minimum speed of movement in the long-term mode at a given power. In each zone of the traction characteristic, different requirements are imposed on the traction electric drive. In the first case, it is necessary to maintain the maximum possible torque according to the coupling conditions, which are constantly changing depending on various factors, both external and internal. The total operating time in the first zone is not large compared to the second zone. The main indicator of performance in this zone is the speed of control of the MEM of the traction 
asynchronous motor, depending on changes in the coupling conditions. The main criterion for the operation of the locomotive traction drive in the first zone of maintaining constant torque is the increased rigidity of the characteristics of the traction engines in the second zone [5]. The main indicator of the efficiency of work in this zone. Unlike the first, the second zone is removed from the coupling restriction curve. So there is no need of high hardness characteristics as when working at the limit of adhesion. Traction and energy characteristics in this zone have a significant impact on energy consumption. Therefore, when working on a hyperbolic characteristic, it is most appropriate to use the control principle to minimize electrical power losses in the inverter-motor system. Stator and rotor variables in the coordinate system $(d-q)$ are represented by their amplitude values and do not contain harmonic components. If the rotor flow coupling vector is oriented along the $\mathrm{d}$ axis, then the projection of the vector, i.e. the frequency of the rotor current at a given flow coupling, determines the electromagnetic moment of the AD. The initial data for the calculation is the specified power of the RAM, the actual speed of the RAM. These parameters determine the required torque of the asynchronous motor

$$
M_{\text {эм }}=\frac{P_{\text {эм }}}{\omega_{\text {М }}}
$$

Then it is compared with the specified limit. If the moment is greater than the limit, the signal is sent to the vector control system. If the moment is less than or equal to the limit, then the signal for the specified moment enters the control system to minimize electrical losses [5]. According to the data of the stator phase current sensors, the frequency of the stator current of the rotor current is determined, which is necessary to determine the magnetic flux. The received data is sent to the minimum power loss unit. When powered by AC power, this system consists of a traction transformer, input $4 \mathrm{q}-\mathrm{S}$ converters, a DC link, and Autonomous voltage inverters. Differential-algebraic equations of processes in power circuits are based on Kirchhoff's laws. They are formed in the following form:

$$
f\left(X_{n+1}, X_{n}, t_{n+1}\right)=0
$$

The solution of equations (1) is performed using the numerical differentiation formula

$$
h\left(X_{n+1}\right)=X_{n+1}=-\frac{1}{\Delta t} \sum_{i=0}^{K} \alpha X_{n+1}
$$

The frequency of the stator current $\omega c$ is determined from the data of the stator phase current sensors. Then, from the difference $\omega r$, and $\omega \mathrm{c}$, the rotor current frequency $\omega r$ is calculated, which is necessary to determine the magnetic flux of the stator.

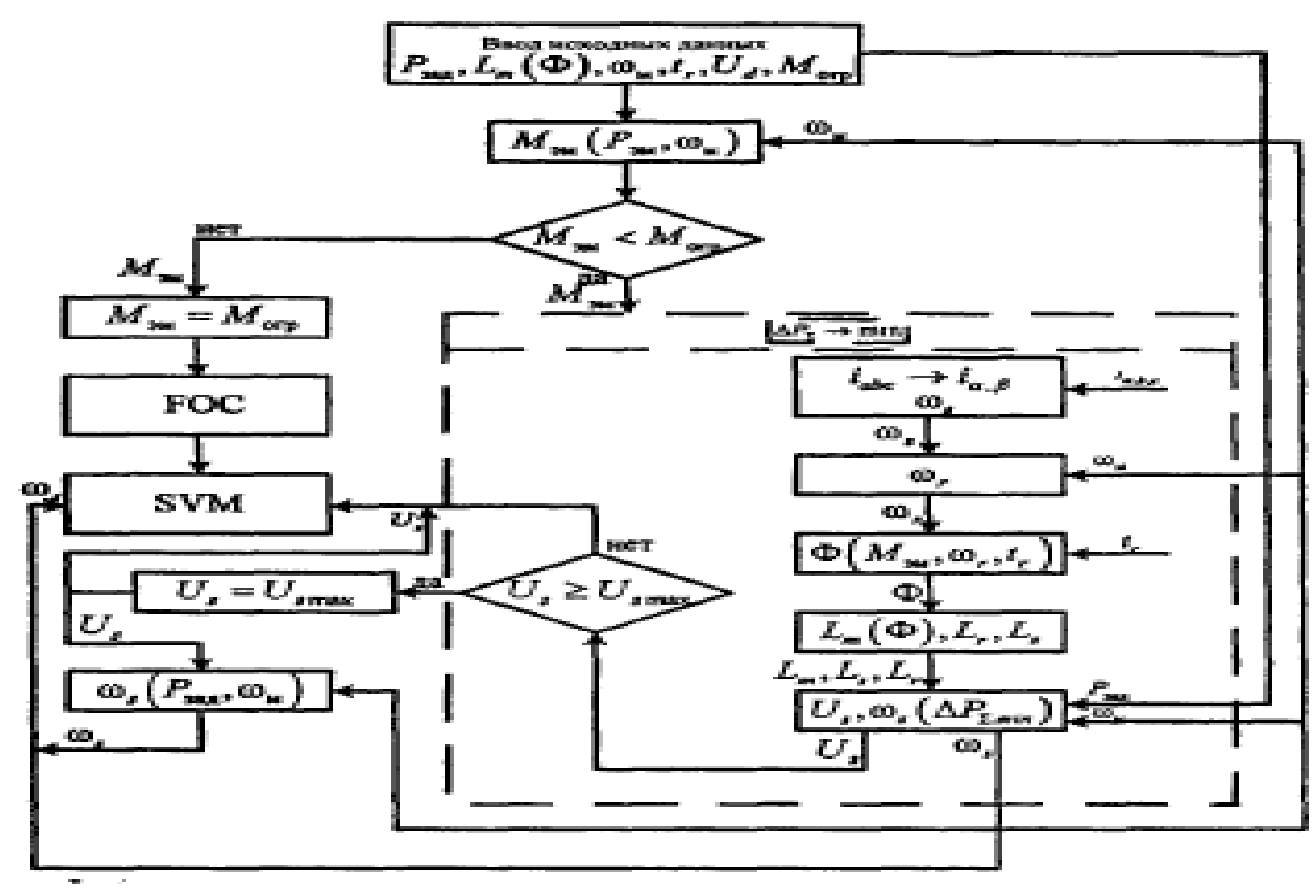

Fig. 2. Algorithm for amplitude-frequency control of an asynchronous traction motor. 


\subsection{Research and Discussions}

Based on the criterion of minimum electrical power loss, from the difference $\omega_{\mathrm{r}}$, and $\omega_{\mathrm{c}}$, the rotor current frequency $\omega_{\mathrm{r}}$ is calculated, which is necessary to determine the magnetic flux of the stator [5].

$$
\Phi=\frac{2 \pi}{\sqrt{34,44 \mathrm{~K} W \mathrm{c}}} \sqrt{\frac{\mathrm{M}\left[R^{2}+\left(\omega \mathrm{L}_{S}\right)\right]}{\mathrm{Z} \omega R}}
$$

The value of the magnetic flux determines the inductance of the magnetization loop LS, which can be used to determine the inductance of the stator LS. the Real Lm (f), taking into account the saturation of steel, is set using the Newton polynomial: To eliminate the dynamic error of adjusting the load angle in dynamic modes, the frequency of the stator current must be formed taking into account its changes:

$$
\omega_{S}=\omega+\omega_{r}+\frac{\partial \delta}{\partial t} \delta
$$

In this case, the effect of changing the highly inertial torque - generating component of the current-the rotor flow coupling-will be eliminated. Then the torque control time will be determined by the speed of the stator current control circuit (adopted by the inertia-free) and the speed of the load angle control circuit. The saturation curve of the magnetic flux is given as a polynomial [5]. :

$$
\begin{aligned}
& \mathrm{L}_{n}=\frac{\Phi}{\Delta \Phi} {\left[\Delta b+\left(\frac{\Phi}{\Delta \Phi}-1\right)\right]\left\{\frac{\Delta \delta}{2}+\left(\frac{\Phi}{\Delta \Phi}\right.\right.} \\
&-2)\left[\frac{\Delta \delta}{6}\left(\frac{\Phi}{\Delta \Phi}-3\right)\left\{\frac{\Phi}{\Delta \Phi}-4\right) \frac{\Delta \delta}{120}\right\}
\end{aligned}
$$

Where: L_(n ), L_ (mo) generalized inductances of the stator and rotor evocation from the optimal frequency of the rotor current in the direction of large values at a constant load moment leads to an increase in the input current of the inverter, and therefore to an increase in the current of the transistors. If the frequency of the rotor current is reduced by increasing the voltage at the specified values of the stator current frequency and the load moment, the input current of the inverter first decreases with increasing phase current, and then increases sharply. A sharp increase in the input current of the inverter at low frequencies of the rotor current is due to saturation of the magnetic circuit of the motor. Based on the data obtained, the minimum power loss is calculated

\section{Conclusion}

Therefore, the improvement of traction and energy indicators of mainline locomotives is possible due to a more complete use of flexible microprocessor control capabilities using rational algorithms that reduce electrical losses in the traction asynchronous drive over the entire operating power range.

\section{References}

1. Dynamically operations in asynchronous tractional gear of mainline electric locomotives. / Y.A. Bakhvalov, G.A. Buzalof, A.A. Zarifyan and others. - M.: Route, 2006.

2. Kirjner D.L. Enforcement demands to the new tractional rolling-stock// Railway transport 2007.- № 8. - C. 13-14.

3. Kolpakhchya P.G., Petrov P.Y.Analysis of the methods on controls of asynchronous tractional engine at electrically propelled vehicles //Informer HEINII. - 2005. - № 2(49). - C. 174-187.

4. Hoshimov, F.A., Bakhadirov, I.I., Erejepov, M., Djumamuratov, B. (2019) Development of method for normalizing electricity consumption E3S Web Conf

139 doi:10.1051/e3sconf/201913901074

5. Burhanhodjaev A.M., Berdiev U.T., Iksar E.V. Eurasian Union of Scientists (ECY) №11 Improvement of traction and energy performance of induction motors №11(68)/ 2019.68 . 